EPJ Web of Conferences 101, 06006 (2015)

DOI: $10.1051 /$ epjconf/ 201510106006

(C) Owned by the authors, published by EDP Sciences, 2015

\title{
KIC 4552982: outbursts and pulsations in the longest-ever pseudo-continuous light curve of a ZZ Ceti
}

\author{
K. J. Bell ${ }^{1, a}$, J. J. Hermes ${ }^{2}$, A. Bischoff-Kim ${ }^{3}$, S. Moorhead ${ }^{1}$, B. G. Castanheira ${ }^{1}$, M. H. Montgomery ${ }^{1}$, \\ and D. E. Winget ${ }^{1}$ \\ 1 Department of Astronomy, University of Texas at Austin, Austin, TX 78712, USA \\ 2 Department of Physics, The University of Warwick, Coventry CV4 7AL, UK \\ 3 Penn State Worthington Scranton, Dunmore, PA 18512, USA
}

\begin{abstract}
KIC 4552982 was the first ZZ Ceti (hydrogen-atmosphere pulsating white dwarf) identified to lie in the Kepler field, resulting in the longest pseudo-continuous light curve ever obtained for this type of variable star. In addition to the pulsations, this light curve exhibits stochastic episodes of brightness enhancement unlike any previously studied white dwarf phenomenon. We briefly highlight the basic outburst and pulsation properties in these proceedings.
\end{abstract}

\section{Introduction}

Around $97 \%$ of Galactic stellar evolution will conclude at the white dwarf stage, and further evolution is essentially a cooling process. When hydrogen-atmosphere white dwarfs cool to a suitable temperature range to support zones of partially ionized hydrogen $(\sim 11,000-12,000 \mathrm{~K}$ for $\log g \sim 8$ [1]), convection drives global nonradial oscillations [2]. These pulsating stars appear photometrically variable as ZZ Cetis. The tools of asteroseismology allow us, through Fourier analysis of time-series photometry, to observationally constrain the extreme conditions of their compact interiors [3-5]. Precision asteroseismology requires extended monitoring of pulsators with limited interruptions. WD J191643.83+393849.7 was identified as a ZZ Ceti in the Kepler field by [6] and was subsequently observed nearly continuously at short (1-minute) cadence for 20 months from Q11 through Q17-a record breaker in this respect. We update the spectroscopically derived parameters from [6] with the 3D corrections of [7] to $T_{\text {eff }}=10,860 \pm 120 \mathrm{~K}$ and $\log g=8.16 \pm 0.06$, corresponding to a white dwarf mass of $0.70 \pm 0.04 M_{\odot}$ [8]. The star has a Kepler magnitude of 17.853 and was assigned the identifier KIC 4552982. The full processed light curve is presented in Figure 1, and the significant regions of its Fourier transform make up Figure 2. A publication detailing our full analysis is nearing completion.

\section{Outbursts}

The immediately striking features of the Kepler light curve in Figure 1 are the occasional excursions to increased brightness throughout the data set. Typical outburst amplitudes are 1-8 \% with durations of 5-10 hours. They occur stochastically in time on a characteristic timescale of 2.5 days.

We have ruled out all conceivable instrumental noise sources and believe these outbursts to be astrophysical in nature. Furthermore, we find difficulty in attributing these outbursts to transient flares from a hidden companion. The flare frequency distribution shows more frequent, highly energetic outbursts than are seen in M dwarf flare stars in the Kepler data set $[9,10]$, and the typical observed

a e-mail: keatonb@astro.as.utexas.edu

This is an Open Access article distributed under the terms of the Creative Commons Attribution License 4.0, which permits unrestricted use, distribution, and reproduction in any medium, provided the original work is properly cited. 


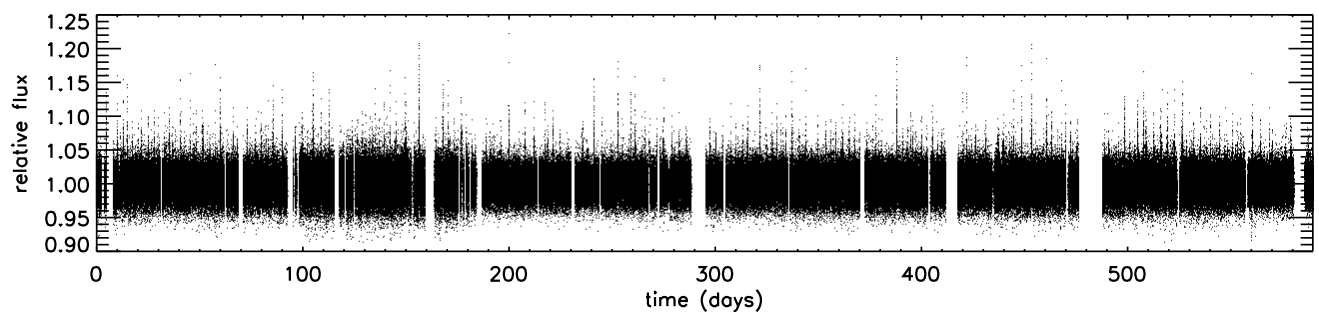

Fig. 1. The full Q11-Q17 Kepler light curve of KIC 4552982.

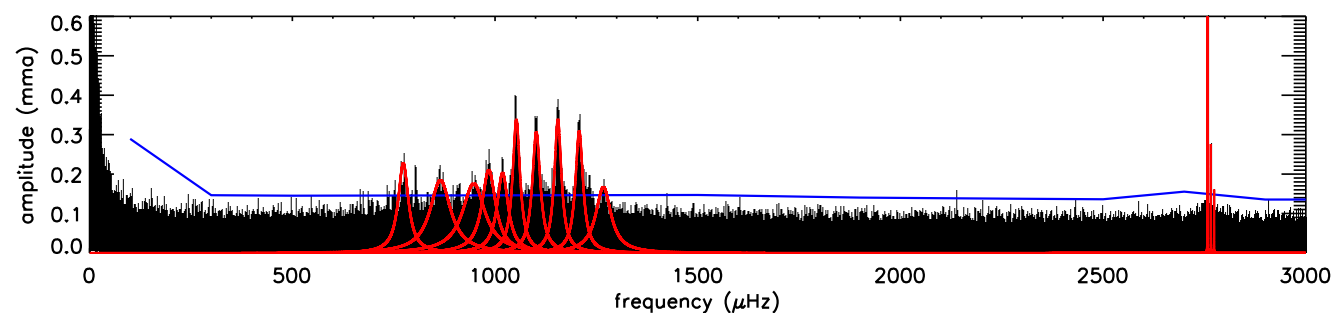

Fig. 2. Fourier transform of the light curve with significant bands of pulsational power and significance threshold.

durations are also in excess of other flare observations. We are left to attribute these outbursts to an as-yet not understood (possibly accretion-related) phenomenon in the white dwarf system or a chance superposition with a highly energetic background system.

\section{Pulsations}

While the pulsation modes are possibly being affected by outbursts in the white dwarf system, the widened power bands in the Fourier transform of the KIC 4552982 light curve (Figure 2) are characteristic of cool ZZ Ceti pulsators with amplitude/phase variations [11-13]. We fit Lorentzian envelopes to the 13 significant power bands to determine pulsation periods. Then we fit these periods to asteroseismic model grids computed from the White Dwarf Evolution Code via the prescription of [14]. With such broad measures of pulsation periods, we can only loosely constrain stellar parameters for now, but we see a preference for higher-mass $\left(\sim 0.7-0.8 M_{\odot}\right)$ models with relatively thick $(\log \sim-4)$ hydrogen layer mass fractions. Interpreting the sharper multiplet surrounding the 361.6-second mode as a $l=1$ rotational triplet gives a rotation period for KIC 4552982 of $\sim 17.4$ hours.

\section{References}

1. Gianninas, A., Bergeron, P., \& Fontaine, G., AJ 132, (2006) 831

2. Brickhill, A. J. MNRAS 251, (1991) 673

3. Winget, D. E., \& Kepler, S. O. ARA\&A 46, (2008) 157

4. Fontaine, G., \& Brassard, P., PASP 120, (2008) 1043

5. Althaus, L. G., Córsico, A. H., Isern, J., \& García-Berro, E., A\&ARv 18, (2010) 471

6. Hermes, J. J., Mullally, F., Østensen, R. H., et al. ApJ 741, (2011) L16

7. Tremblay, P.-E., Ludwig, H.-G., Steffen, M., \& Freytag, B. A\&A 559, (2013) A104

8. Wood, M. A., White Dwarfs 443, (1995) 41 Barclay, T., Fanelli, M., et al., PASP 124, (2012) 963

9. Hawley, S. L., Davenport, J. R. A., Kowalski, A. F., et al., ApJ 797, (2014) 121

10. Davenport, J. R. A., Hawley, S. L., Hebb, L., et al., ApJ 797, (2014) 122

11. Pfeiffer, B., Vauclair, G., Dolez, N., et al., A\&A 314, (1996) 182

12. Kleinman, S. J., Nather, R. E., Winget, D. E., et al., ApJ 495, (1998) 424

13. Hermes, J. J., Charpinet, S., Barclay, T., et al., ApJ 789, (2014) 85

14. Bischoff-Kim, A., Montgomery, M. H., \& Winget, D. E. ApJ 675, (2008) 1505 